RE: Editorial, Vol. 65, Issue 3, Fall 2018, "When Can the Recovery Process Be Delegated to Staff?"

\section{Dear Dr. Ganzberg:}

Your editorial is unfortunately posttimely. Some dentist, predominantly oral and maxillofacial surgeon (OMS), "advisors" have already, and are currently, convincing regulators that a dental assistant (presumably a high school graduate, but this does not seem to be required) can be taught the art and science of anesthesiology by taking an online course of 36 clockhours' duration, if she or he has worked for an oral surgeon for 6 months and is currently certified in BLS. These same assistants can then be "certified" by passing an examination, the Dental Anesthesia Assistant $\mathrm{Na}-$ tional Certification Examination, of 100 questions. The misconception of the lay patient/consumer and dental boards is that since these "adequately trained" dental assistants can competently monitor a patient under general anesthesia and in some states administer anesthesia medications, then it follows that "assuredly, they can recover a patient from general anesthesia." This certification is nothing more than a false credential granted to lay persons, to fool unsuspecting patients, Medicaid and other insurance companies, and dental boards for one purpose only: to enable OMSs (almost exclusively) to uniquely double bill for one professional providing both surgery and anesthesia services, rather than two, as occurs everywhere else in the world.

There are 4 stages to rendering anesthesia care: stage 1, preoperative evaluation and patient preparation; stage 2, operating area for provision of anesthesia services; stage 3, postanesthesia recovery, in which the patient may still be significantly under the influence of anesthetic agents (in hospitals with a dedicated postanesthesia care unit); and stage 4, postrecovery holding (usually the same area as the preop holding in surgery centers). In most dental facilities, stages 2 and 3 are completed in the same area. Stage 4 is often a different area to reunite the family with the patient and to separate postanesthesia patients from patients awaiting surgery. Some dentists may choose to separate the postanesthesia care unit phase (stage 3) from the operating phase (stage 2), but supplies, equipment, and qualified licensed professionals, such as registered nurses with anesthetic recovery experience, must be present for proper patient evaluation and intervention wherever that location is.

Recovery begins at the very moment the last drug enters the body, not when the surgery is completed. Initial recovery (stage 3) may be completed in the operating suite with the anesthesia provider continuously present until transfer to a postrecovery holding area. Once safe mobilization occurs by whatever means, the patient is then discharged from the facility. Only appropriately credentialed and licensed individuals (experienced anesthesia recovery registered nurses or independent anesthesia providers) are acceptable personnel to perform recovery duties, that is, interpret monitoring data; perform patient evaluation/diagnosis; perform definitive care and implementation of verbal/ written orders, including the administration of vasoactive substances used for blood pressure and heart rate extremes; treatment of laryngospasm, such as the administration of succinylcholine which will cause temporary paralysis necessitating ventilation support and possible intubation; and a whole host of other resuscitation drugs and procedures.

The integrity of the profession of dentistry is at extreme risk, if not already damaged. There are those willing to eliminate professionals from providing care but still charge for those services as though they were truly provided by such professionals. This antiquated paradigm is void of veracity and currently a subject of significant concern with other professional dental and medical groups.

Joe Seay, DDS, MS

Dentist Anesthesiologist Specialist in Anesthesiology 